\title{
Adverse outcomes in anticoagulated patients undergoing percutaneous left atrial appendage ligation
}

\author{
Anetta Undas ${ }^{1,2}$ \\ ${ }^{1}$ Department of Cardiac Surgery, Anesthesiology and Experimental Cardiology, Institute of Cardiology, Jagiellonian University \\ School of Medicine, Krakow, Poland \\ ${ }^{2}$ Krakow Centre for Medical Research and Technologies, John Paul II Hospital, Krakow, Poland
}

Adv Interv Cardiol 2020; 16, 2 (60): 231-232

DOI: https://doi.org/10.5114/aic.2020.96075

Percutaneous left atrial appendage (LAA) suture ligation using the LARIAT (SentreHEART Inc, Redwood, CA) device in patients with atrial fibrillation (AF) for the prevention of thromboembolic events was introduced in 2009. The Polish recommendations regarding LAA closure published in 2018 also presented this uncommonly used surgical technology to occlude the LAA in high-risk AF patients [1]. I read with keen interest the article by Litwinowicz et al. in the latest issue of Advances in Intervential Cardiology, in which the authors reported clinical outcomes in 139 patients following the use of the LARIAT device to close the LAA from December 2009 to December 2010 [2]. A key finding of the study is no difference in rates of thromboembolic and bleeding events as well as mortality between anticoagulated and non-anticoagulated AF patients during up to 60 months of follow-up. The former group involved 75 of the 139 patients, including $40 \%$ of anticoagulated subjects on a vitamin $\mathrm{K}$ antagonist (VKA) and $18 \%$ on non-VKA oral antagonists (NOAC) at the end of follow-up [2]. Apart from a rather surprisingly large proportion of AF patients in whom the procedure was performed with continued anticoagulation, which had been previously assessed as contraindicated or ineffective, it would be interesting to provide data on the indication for LAA closure and quality of anticoagulation with VKA. Three thromboembolic arterial events were observed on VKA, which suggests poor quality of anticoagulant therapy. This speculation could be supported by the fact that a single major bleeding event was observed on VKA during follow-up, which is a rather uncommon finding in patients on VKA. The current study could also be considered as an additional indirect suggestion to widely use NOAC in AF patients as recommended by most experts especially in AF patients at high thrombo- embolic and bleeding risk, similar to the present patient population [3]. From a practical point of view, it would be of interest to provide more data on the subsequent therapeutic strategy after the adverse events reported here. Were the patients with adverse events switched to other treatment strategies? Were patients after severe bleeding off anticoagulation? It is unclear whether the clinical outcomes could be affected by patients' age as the most relevant risk factor for both thromboembolism and bleeding in AF [3]. In a previous study, Litwinowicz et al. [4] found that during long-term follow-up thromboembolic risk after LAA occlusion was not related to this potent stroke risk factor. Did the authors look at a composite of efficacy and safety endpoints to determine whether age affects the study results?

Taken together, the study by Litwinowicz et al. [2] presented unique follow-up data on AF patients following LAA occlusion with the LARIAT device, which underscore the need for appropriate decision-making to balance the risk and benefits from the procedure in high-risk patients, followed by either long-term anticoagulation, preferably with NOACs, or not.

\section{Conflict of interest}

The author declares no conflict of interest.

\section{References}

1. Grygier M, Wojakowski W, Smolka G, et al. Left atrial appendage occlusion: consensus document of Association of Cardiovascular Interventions and Heart Rhythm Section of Polish Cardiac Society. Kardiol Pol 2018; 76: 677-97.

2. Litwinowicz R, Filip G, Sobczyk D, et al. Long-term effect of anticoagulation following left atrial appendage occlusion with the LARIAT device in patients with nonvalvular atrial fibrillation:

\section{Corresponding author:}

Anetta Undas MD, PhD, Department of Cardiac Surgery, Anesthesiology and Experimental Cardiology, Institute of Cardiology, Jagiellonian University Medical College, 80 Pradnicka St, 31-202 Krakow, Poland, phone: +48 126143004 , fax: +48 12 614 2120 , e-mail: mmundas@cyf-kr.edu.pl

Received: 7.05.2020, accepted: 7.05.2020. 
impact on thromboembolism, bleeding and mortality. Real life data. Adv Interv Cardiol 2020; 16: 89-96.

3. Balsam P, Ozierański K, Tymińska A, et al. Comparison of clinical characteristics of real-life atrial fibrillation patients treated with vitamin $\mathrm{K}$ antagonists, dabigatran, and rivaroxaban: results from the CRAFT study. Kardiol Pol 2018; 76: 889-98.

4. Litwinowicz R, Bartuś M, Ceranowicz P, et al. Stroke risk reduction after left atrial appendage occlusion in elderly patients with atrial fibrillation: long term results. Pol Arch Intern Med 2018; 128: 327-9. 